\title{
Analysis of endometrial microbiota in intrauterine adhesion by high-throughput sequencing
}

\author{
Tianmei Qiu ${ }^{1 \#}$, Lei Liu ${ }^{2 \#}$, Hongwei Zhou ${ }^{3}$, Huafang Sheng ${ }^{3}$, Yuanli He ${ }^{1}$, Mubiao Liu $^{2}$, Huihua Cai ${ }^{4}$ \\ ${ }^{1}$ Department of Obstetrics and Gynecology, Zhujiang Hospital, Southern Medical University, Guangzhou, China; ${ }^{2}$ Department of Obstetrics and \\ Gynecology, Guangdong Provincial People's Hospital, Guangdong Academy of Medical Sciences, Guangzhou, China; ${ }^{3}$ State Key Laboratory of \\ Organ Failure Research, Division of Laboratory Medicine, Zhujiang Hospital, Southern Medical University, Guangzhou, China; ${ }^{4}$ Department \\ of Gynecology, Affiliated Foshan Maternity \& Child Healthcare Hospital, Southern Medical University (Foshan Maternity \& Child Healthcare \\ Hospital), Foshan, China \\ Contributions: (I) Conception and design: H Cai, M Liu, Y He; (II) Administrative support: Y He, M Liu; (III) Provision of study materials or patients: \\ Y He, H Zhou, H Sheng; (IV) Collection and assembly of data: T Qiu, L Liu; (V) Data analysis and interpretation: T Qiu, H Cai, H Zhou, H Sheng; \\ (VI) Manuscript writing: All authors; (VII) Final approval of manuscript: All authors. \\ \#These authors contributed equally to this work. \\ Correspondence to: Huihua Cai. Department of Gynecology, Affiliated Foshan Maternity \& Child Healthcare Hospital, Southern Medical University \\ (Foshan Maternity \& Child Healthcare Hospital), NO. 11, Renminxi Road, Foshan 528000, Chinai. Email: chh2004@126.com; Mubiao Liu. \\ Department of Obstetrics and Gynecology, Guangdong Provincial People's Hospital, Guangdong Academy of Medical Sciences, NO. 106, \\ Zhongshan Road, Guangzhou 510080, China. Email: liumb1972@126.com; Yuanli He. Department of Obstetrics and Gynecology, Zhujiang \\ Hospital, Southern Medical University, 253 Industrial Avenue Middle, Guangzhou 510282, China. Email: heyuanli310@163.com.
}

Background: Intrauterine adhesions (IUA) arise from scar tissue formation between the endometrial surfaces in response to mechanical or infectious injuries. However, the potential role of endometrial microbiota in IUA remains unclear. We aimed to explore the composition of endometrial microbiota and its potential role in IUA.

Methods: We retrospectively enrolled 46 patients diagnosed with IUA and 21 infertility patients without intrauterine lesions, as control subjects. All cases were diagnosed with hysteroscopy and endometrial tissues were taken from the intrauterine cavity using a hysteroscopic cutting ring without electricity study. After endometrial samples were collected, DNA was extracted and amplified for barcoded Illumina highthroughput next-generation sequencing targeted to the $16 \mathrm{~S}$ rRNA V4 region for microbiota. Microbiota data were compared between two groups using $\alpha$-diversity, $\beta$-diversity and Nonmetric Multidimensional Scaling based on Weighted Unifrac distance.

Results: At the phyla level, the dominant bacteria included Proteobacteria, Firmicutes, Bacteroidetes and Actinobacteria. Proteobacteria accounted for more than $64.48 \%$. At the genus level, the proportions of Klebsiella, Shewanella, and Lactobacillus were higher in patients with IUA than in non- IUA participants (20.67\% and 8.77\%, $\mathrm{P}=0.006,13.37 \%$ and $4.53 \%, \mathrm{P}=0.175,12.74 \%$ and $6.95 \%, \mathrm{P}=0.882$; respectively). The proportion of Acinetobacter was significantly lower in patients with IUA than in non- IUA participants $(\mathrm{P}=0.005)$.

Conclusions: Endometrial microbiota differ between patients with IUA and infertility patients without intrauterine lesions, and the potential variation of endometrial microbiota might cause IUA.

Keywords: Intrauterine adhesions (IUA); endometrial microbiota; 16S rRNA

Submitted Mar 24, 2020. Accepted for publication Nov 22, 2020.

doi: 10.21037/atm-20-2813

View this article at: http://dx.doi.org/10.21037/atm-20-2813 


\section{Introduction}

Intrauterine adhesions (IUA), also known as Asherman's syndrome, is an intrauterine condition characterized by the physiological endometrium being replaced by fibrotic tissue or scar after the mechanical or infectious injury of the endometrium $(1,2)$. IUA could progressively cause hypomenorrhea, amenorrhea, infertility, spontaneous abortions, and even placenta implantation. Recently, the rate of IUA recurrence, especially in severe cases, remains high even after hysteroscopic adhesiolysis combined with various adjuvant therapies. Therefore, IUA therapy is still challenging (3). Endometrial damage and infection are recognized as the two major risk factors of IUA.

The balance of micro-ecology in the female reproductive tract plays a key role in health. An increasing body of evidence suggests that the change of composition and distribution of endometrial microbiota is related to endometrial diseases such as endometrial polyps, endometrial cancer, and infertility (4-6). Patients with IUA have micro-ecological imbalance in the lower genital tract, and the V4 region or the V3 and V4 region of the $16 \mathrm{~S}$ rDNA in each sample was amplified by PCR method $(7,8)$. However, there are few reports on endometrial microbiota in patients with IUA.

Moreover, a growing body of evidence shows that organ fibrosis is associated with microbiota. The specific microbial signatures can be used to distinguish the severity of liver diseases, such as mild disease and advanced fibrosis (9). Recent 454 pyrosequencing data have revealed that the progression of idiopathic pulmonary fibrosis is related to the presence of specific members in Staphylococcus and Streptococcus genera, suggestive of the role of microbiome in fibrotic processes (10).

Given the inflammatory profile and fibrosis essence of IUA, in this study we used high throughput sequencing techniques to characterize endometrial microbial communities in patients with IUA and the intrauterine microbial diversity difference compared to the females without intrauterine lesions. The authors present the study in accordance with the MDAR reporting checklist (available at http://dx.doi.org/10.21037/atm-20-2813).

\section{Methods}

\section{Patients}

This study was conducted in accordance with the Declaration of Helsinki (as was revised in 2013). The study was approved by the Medical Ethics Committees at the Zhujiang Hospital of Southern Medical University (NO. 2019-KY-077-01) and all the patients provided informed consent. Demographic data of 67 participants from March 2016 to March 2019 were collected retrospectively. Among them, 46 patients with IUA were enrolled as the observation group (Group IUA), and 21 infertile females without intrauterine lesion were enrolled as the control group (Group C). Subjects eligible for Group IUA met the following criterion: diagnostic hysteroscopy confirmed the presence of adhesions in the intrauterine cavity. The inclusion criteria in Group $\mathrm{C}$ were the following: hysteroscopy and subsequent endometrial pathology excluded the lesions in the intrauterine cavity. The exclusion criteria of all participants were: women who had taken antibiotics within three weeks pre-operation, other intrauterine lesions such as endometrial polyps, submucosal myoma, endometrial cancer and endometrial hyperplasia, coagulopathy, vaginitis and acute pelvic inflammatory disease. Group IUA was further divided into three subgroups based on American Fertility Society Rating System: 20 patients scored from 1 to 4 were classified as Stage I (Group IUA-Mild), and 12 patients scored from 5 to 8 as Stage II (Group IUA-Moderate), and the remaining scored from 9 to 12 as Stage III (Group IUA-Severe). As shown in Table 1, clinical characteristics of these groups were comparable except for the number of intrauterine operations.

\section{Samples}

All samples were collected in the early stage of endometrial proliferation, or any day for the patients with secondary amenorrhea. After vaginal and cervical canal disinfection, endometrial tissues were taken gently from the intrauterine cavity using a hysteroscopic cutting ring without electricity. During the collection of endometrial samples, hysteroscope sheath was used to avoid contamination by vaginal microbiota. Each sample was placed in a sterile tube and immediately stored in liquid nitrogen for DNA extraction.

\section{Genomic DNA extraction, bacterial $16 S r R N A$ amplification and high-throughput sequencing}

Genomic DNA was extracted from endometrial tissues using HiPure Bacterial DNA Kit (Magen, USA). The concentration and quality of purified DNA were determined by a spectrophotometer at 230 and 260 
Table 1 Clinical characteristics of women enrolled in the study

\begin{tabular}{lcccc}
\hline Items & Group IUA-H & Group IUA-M & Group IUA-L & Group C \\
\hline Age (years) & $29.79 \pm 1.01$ & $30.58 \pm 1.25$ & $31.85 \pm 1.45$ & $33.14 \pm 1.02$ \\
Gravidity & $2.64 \pm 0.39$ & $2.17 \pm 0.32$ & $2.90 \pm 0.35$ & $1.95 \pm 0.15$ \\
Parity & $0.71 \pm 0.83$ & $0.25 \pm 0.13$ & $0.85 \pm 0.18$ & $0.85 \pm 0.25$ \\
No. of abortions/curettage & $2.00 \pm 1.24$ & $2.00 \pm 0.28$ & $2.25 \pm 0.33$ & $1.01 \pm 0.25$ \\
\hline
\end{tabular}

Group IUA-H, intrauterine adhesion patients with high-grade. Group IUA-M, intrauterine adhesion patients with middle-grade. Group IUA-L, intrauterine adhesion patients with low-grade. Group C, the control group. Mean \pm SE are shown.

nm (NanoDrop-One, Thermo Fisher Scientific, MA, USA). The V4 region of the $16 \mathrm{~S}$ rDNA was amplified using Premix-Taq (Takara Biotechnology, Dalian, China), and $515 \mathrm{~F} / 806 \mathrm{R}$ primers with $12 \mathrm{bp}$ barcode (515F, 5'-GTGCCAGCMGCCGCGGTAA-3'; 806R, 5'-GGACTACHVGGGTWTCTAAT-3'). The cycling conditions for PCR were as follows: $94{ }^{\circ} \mathrm{C}$ for $5 \mathrm{~min}$, followed by 30 cycles of $94{ }^{\circ} \mathrm{C}$ for $30 \mathrm{sec}, 52{ }^{\circ} \mathrm{C}$ for $30 \mathrm{sec}$, $72{ }^{\circ} \mathrm{C}$ for $30 \mathrm{sec}$ and another $10 \mathrm{~min}$ at $72{ }^{\circ} \mathrm{C}$. According to Gene Tools Analysis software (version 4.03.05.0, SynGene), PCR products were mixed into one tube in equal proportions, and the amplicon mixture was purified with E.Z.N.A. Gel Extraction Kit (Omega, USA). The 16S rDNA sequencing was performed with a high-throughput next-generation Illumina Hiseq2500 platform (11).

\section{DNA sequence data analysis and taxonomy}

The quality filtering on the Paired-end reads from the original DNA fragments was processed with Trimmomatic software (12) (version 0.33, http://www.usadellab.org/ $\mathrm{cms} /$ ? page=trimmomatic) to remove paired-end reads with one or more ambiguous bases. The retained pairedend reads were trimmed at the 3 ' end to eliminate the continuous bases with a quality score $<20$ and reads with sequence length $<100$ bp to obtain high-quality reads. Paired-end clean reads were merged using FLASH (version 1.2.11, https://ccb.jhu.edu/software/FLASH/) according to the relationship of the overlap between the paired-end reads. The sequences were assigned to each sample based on their unique barcode and primer using Mothur software (13) (version 1.35.1, http://www.mothur.org), after which the barcodes and primers were removed to get effective Clean Tags.

Ultimately, an average of $158,737.64 \pm 112,78.96$ reads per sample with a total of $10,635,422$ sequence reads $(47,952-$ 421,485 reads per sample) (Table S1) were obtained after quality control. Using the usearch software (14) (version 10, http://www.drive5.com/usearch/), the sequences with $97 \%$ or higher similarity were grouped into the same operational taxonomic units (OTU).

Further processing was performed using QIIME (15). The taxonomic assignment of representative sequences was performed using the Ribosomal Database Project (RDP) Classifier (16) and a minimum confidence threshold to default was adjusted to $50 \%$. Dominance and the Simpson indexes were used to measure $\alpha$-diversity for species richness and evenness within different bacterial populations. The weighted UniFrac (17) was used to measure $\beta$-diversity for the diversity between bacterial communities in terms of ecological distance between samples. The difference in bacterial community composition was analyzed by Nonmetric Multidimensional Scaling (NMDS) based on Weighted Unifrac distance. Moreover, the difference in $\beta$-diversity among groups was analyzed by ADONIS, a multivariate analysis of variance based on distance matrices and permutation.

\section{Statistical analysis}

Statistical analysis was performed with Statistical Product and Service Solutions (SPSS) (version 20.0). Data normality was tested with the Kolmogorov-Smirnov test. Homogeneity of variance was detected with the Levene test. Data were presented as means \pm standard deviations. Data were compared by the Mann-Whitney test or the KruskalWallis analysis of variance on ranks, followed by Dunn's test. The statistical significance was set at two-side $\mathrm{P}<0.05$.

\section{Results}

\section{Sequencing coverage}

To characterize the different bacteria population in endometrial tissues, we performed deep sequencing of the 

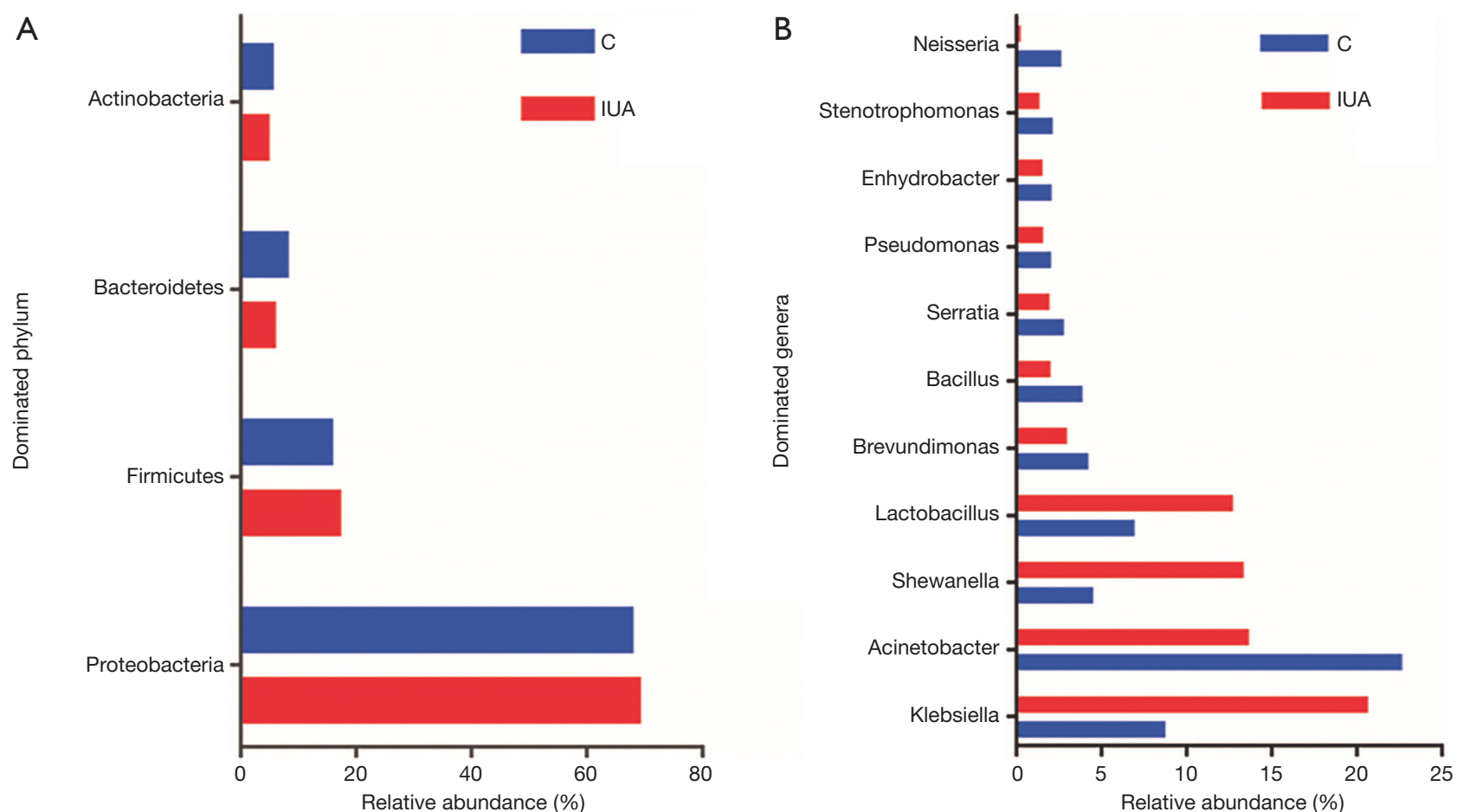

Figure $116 \mathrm{~S}$ rRNA gene analysis revealed taxonomic variations and high diversity of endometrial microbiota between patients with IUA and infertile females without intrauterine lesion. (A) Bars plots showed the relative abundance of the 4 most abundant bacterial phyla in two groups. (B) Bars plots showed the relative abundance of the 10 most abundant bacterial groups at the genus level in two groups.

16S rRNA V4 region of all 67 samples. In total, 10,635,422 filtered clean reads $(158,737.64 \mathrm{reads} / \mathrm{sample})$ and $10,089,655$ filtered clean tags $(150,591.87$ reads/sample) were obtained from all samples (Table S1). A total of 1,163 OTUs were identified from all samples.

\section{Differences in bacterial population between Group IUA and Group $C$}

The most abundant phylum in endometrium of Group C and Group IUA was Proteobacteria, which accounted for $68.11 \%$ and $69.36 \%$, respectively, followed by Firmicutes (16.01\% and $17.47 \%)$, Bacteroidetes $(8.38 \%$ and $6.15 \%)$ and Actinobacteria (5.73\% and 5.00\%). There was no difference in the proportion between the two groups $(\mathrm{P}>0.05$, Figure $1 A)$. At the genus level (Figure 1B), bacterial communities in Group C were dominated by Acinetobacter (22.68\%), followed by Klebsiella (8.77\%), Lactobacillus (6.95\%) and Shewanella (4.53\%). However, bacterial communities in Group IUA were dominated by Klebsiella (20.67\%), Acinetobacter (13.67\%), Shewanella (13.37\%) and Lactobacillus (12.74\%).
The proportion of Acinetobacter was significantly lower in Group IUA than in Group C ( $\mathrm{P}=0.005)$, while the proportion of Klebsiella was significantly higher in Group IUA than in Group C $(\mathrm{P}=0.006)$. The rarefaction curves of Simpson index showed that $\alpha$-diversity in Group IUA was significantly lower than that in Group C $(\mathrm{P}=0.01)$ (Figure $\mathrm{S} 1 \mathrm{~A})$, while Group IUA had more OTUs than Group C $(\mathrm{P}=0.257)$ (Figure S1B). In addition, $\beta$-diversity analysis revealed the difference in microbiota structure. Endometrial microbiota showed significant difference in Group IUA and Group C $(\mathrm{P}=0.048$, Figure $2 A)$.

\section{Overall microbiota structure difference among four groups}

There was no difference in the observed species between the groups $(\mathrm{P}=0.491$, Figure $\mathrm{S} 2)$. As shown in the Figure S2, rarefaction curves of Simpson index indicated significant difference in $\alpha$-diversity of endometrial bacteria between the different stage of IUA and Group C $(\mathrm{P}=0.011)$. Group $\mathrm{C}$ had the highest $\alpha$-diversity of endometrial bacteria. Both endometrial $\alpha$-diversity in Group IUA-Mild and 

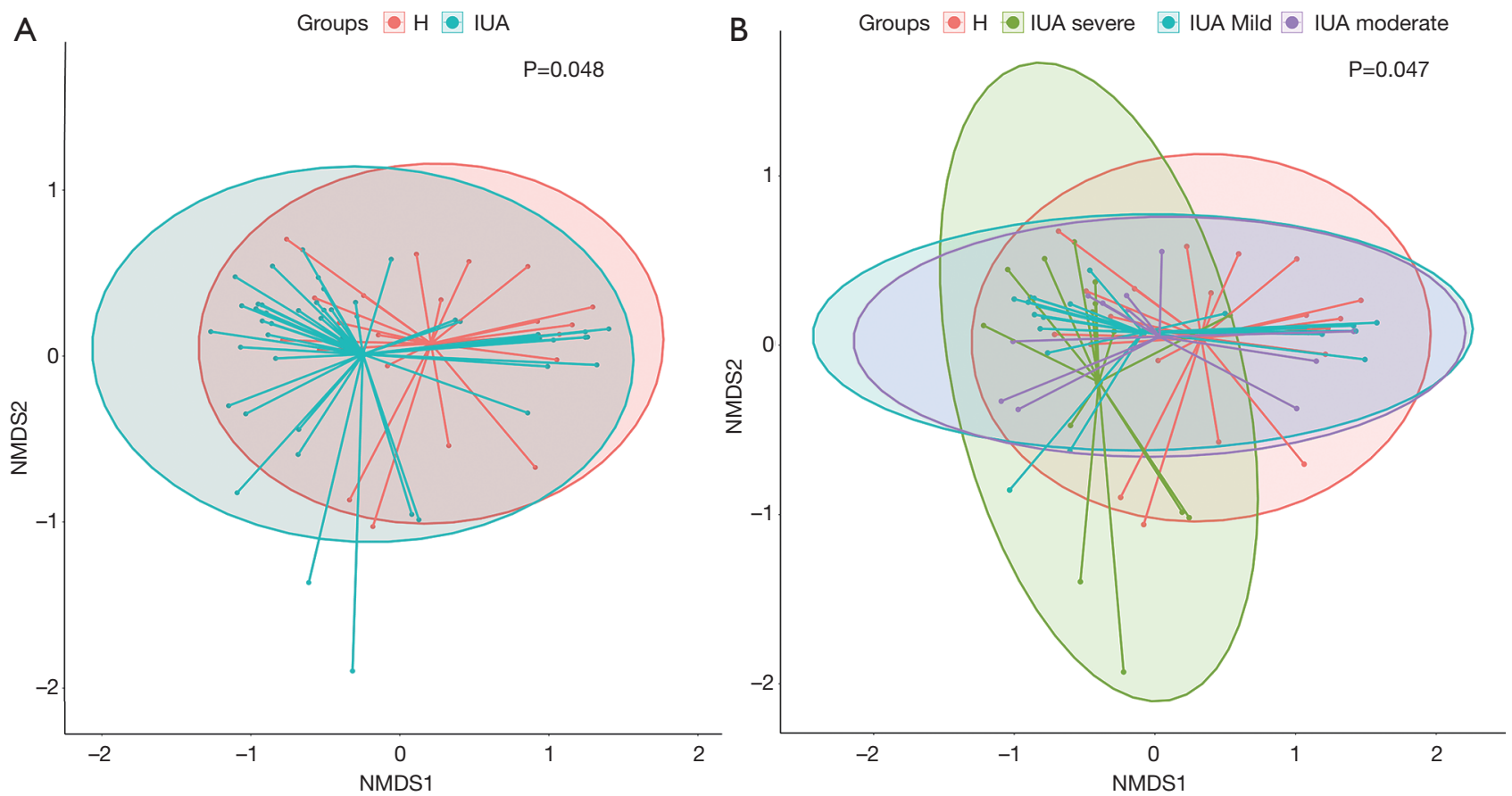

Figure 2 Ordination plot based on weighted UniFrac distance showed the relationship between different disease states. Each point represented a sample. The red color represented infertile females without intrauterine lesion, the green color represented patients with mild IUA, the purple color represented patients with moderate IUA, and the yellow color represented patients with moderate IUA. (A) The endometrial microbiota was significantly different between patients with IUA and infertile females without intrauterine lesion $(\mathrm{P}=0.048)$. $(\mathrm{B})$ The endometrial microbiota showed difference between patients with mild IUA and infertile females without intrauterine lesion $(\mathrm{P}=0.022)$.

Group IUA-Severe were significantly different from that in Group $\mathrm{C}(\mathrm{P}=0.012, \mathrm{P}=0.002)$. No difference in $\alpha$-diversity was found between Group IUA-Mild and group IUAModerate or Group IUA-Severe, respectively ( $\mathrm{P}=0.090$, $\mathrm{P}=0.512)$. There was significant difference between Group IUA-Moderate and Group IUA-Severe $(\mathrm{P}=0.046)$, but not between Group IUA-Moderate and Group C $(\mathrm{P}=0.811)$. The UniFrac-based nonmetric Multidimensional Scaling was carried out to explore the difference in bacterial community composition between groups. As shown in Figure $2 B$, consistent with the $\alpha$-diversity analysis, there was significant difference in endometrial $\beta$-diversity among four cohorts $(\mathrm{P}=0.047)$. In addition, endometrial microbiome of Group IUA-Mild was different from that of Group C $(\mathrm{P}=0.022)$, but endometrial microbiome of Group IUAModerate and Group IUA-Severe were not different from that of Group C, and there was no difference in $\beta$-diversity among the three IUA subgroups. These results indicated a relationship between IUA and the variation of endometrial microbiota.

\section{Bacterial composition and community structure in four groups}

As shown in Figure 3A, at the phylum level, all groups were dominated by Proteobacteria, Firmicutes, Bacteroidetes and Actinobacteria. With the increase of the severity of adhesions, the proportion of Proteobacteria decreased $(73.30 \%, 70.29 \%$ and $64.48 \%$ ), while the proportion of Firmicutes increased (12.79\%, $14.02 \%$ and $25.58 \%)$, although there was no difference. As shown in Figure 3B, compared with Group $\mathrm{C}$, the abundance of Klebsiella sequences was significantly higher in endometrial communities of Groups IUAmild, IUA-moderate and IUA-severe (31.44\%, 12.51\% and $18.06 \%, \mathrm{P}=0.046$ ), while Acinetobacter sequences was significantly lower $(12.74 \%, 16.18 \%$ and $12.10 \%, \mathrm{P}=0.020)$. In addition, Group IUA-Mild had the highest proportion of Lactobacillus.

\section{Discussion}

With the development of high-throughput sequencing 

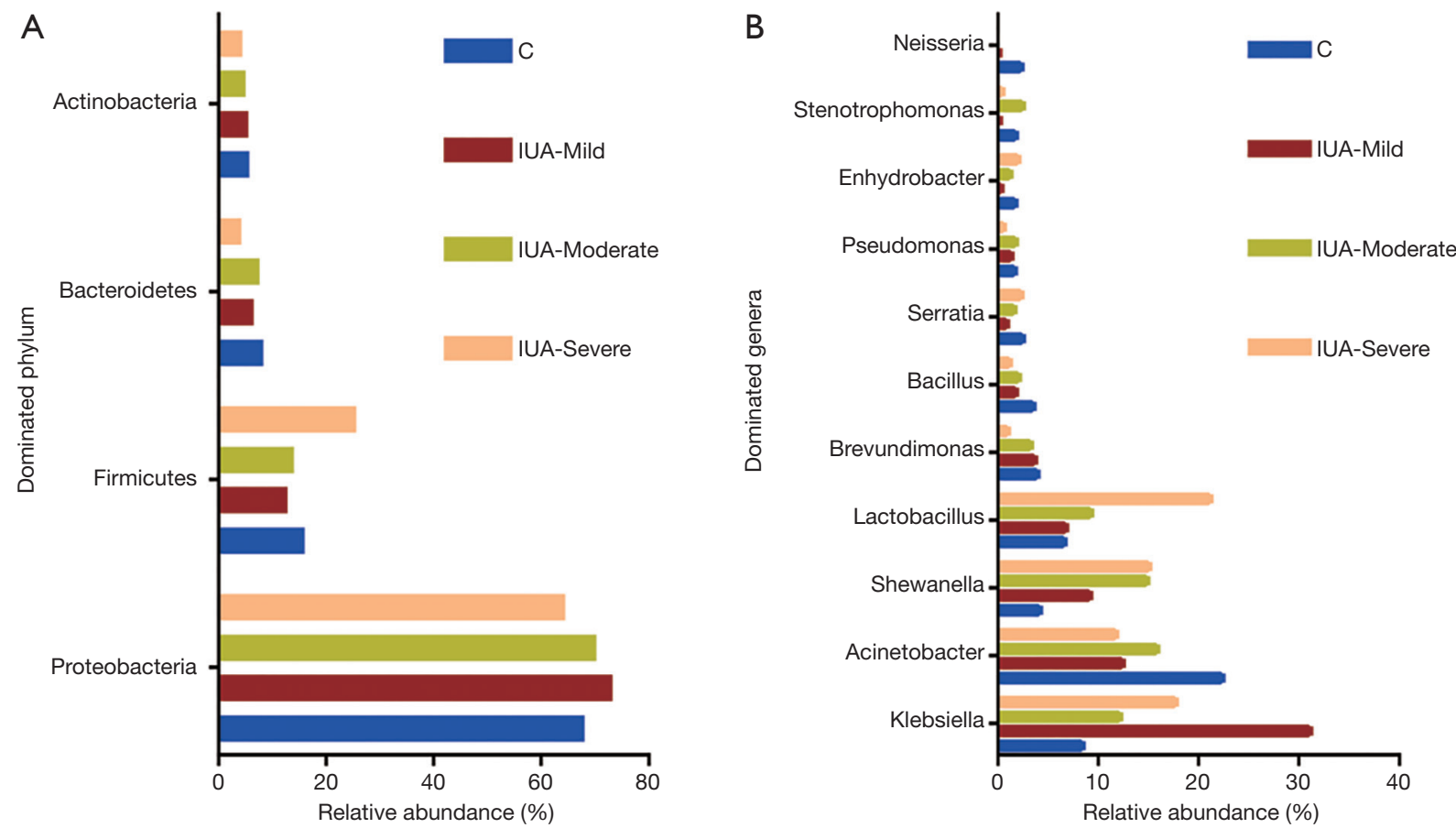

Figure 3 16S rRNA gene analysis showed taxonomic variations and high diversity of endometrial microbiota among patients with three IUA subgroups and infertile females without intrauterine lesion. (A) Bars plots showed the relative abundance of the 4 most abundant bacterial phyla in four groups. (B) Bars plots showed the relative abundance of the 10 most abundant bacterial groups at the genus level in four groups.

technology, some uncultivable, low abundant and unclassified microorganisms in the upper reproductive tract have been recognized. Recent evidence has revealed the association between the microbiota in the vagina or cervical canal and IUA $(7,8)$. Up to now, there is few report on the structure and distribution of endometrial microbiota in patients with IUA. Here, we provided the first evidence that endometrial microbiota in patients with IUA were different from those in infertility patients without intrauterine lesions, and the potential variation of endometrial microbiota might be related with the occurrence of IUA.

In present study, for all participants, the genera with relatively high abundance of endometrial bacteria were Proteobacteria, Firmicutes, Bacteroidetes, Actinobacteria and Lactobacillus. These results are consistent with previous reports on uterine microbiota in patients with endometrial polyps, endometrial cancer, and infertility (4-6). Klebsiella, Acinetobacter, Shewanella, Brevundimonas, Bacillus, Serratia, Pseudomonas, Stenotrophomonas, Neisseria, were also detected in all participants. However, our results are not consistent with some results reported previously $(7,8)$. Liu et al. (7) showed that patients with IUA had significantly lower percentage of Firmicutes and higher percentage of Actinobacteria in the vagina, and half of the patients had overgrowth of Gardnerella and Prevotella accompanied with the reduction of Lactobacillus in the vagina. Zhao et al. (8) demonstrated that the proportion of Firmicutes was higher in vagina and cervical canal from most cases with IUA, but some species including Acidobacteria, Euryarchaeota, Chlamydia, Chlorobi, Planctomycetes and TM6 (Dependentiae) almost disappeared. We found that the proportion of Actinobacteria was lower than that of Firmicutes, while Lactobacillus increased among endometrial microbiota in patients with IUA. This discrepancy might be related to different severity of IUA, different sites of samples and different ecological niches in the reproductive tract.

For the structure of the microbial community, mild IUA cohort could be noticeably distinguished from the control cohort. This indicates that the microbiota may play a role in the early stage of endometrial fibrosis. However, the structural difference was not significant between the moderate or severe IUA cohort and the mild IUA 
cohort, indicating differential microbial community of endometrium associated with the severity of IUA.

For the distribution of endometrial microbiota, the uniformity of mild IUA cohort was the lowest, and the overall uniformity of all IUA cohorts was still lower than that of the control cohort. There may be a period of transient microflora disturbance, and then the endometrial microbiota would be renormalized to a new equilibrium state.

In addition, the number of endometrial Klebsiella in patients with IUA was significantly higher than that of the control group, and this bacterium was mainly enriched in the mild IUA cohort. Klebsiella is a kind of encapsulated Gram-negative bacilli in Enterobacteriaceae, and it is a typical conditional pathogen (18). Some strains of Klebsiella can produce virulence factors such as lipopolysaccharide (LPS), which acts on the receptor Toll-like receptors 4 (TLR4) to induce fibrosis and inflammation (19). As we know, the expression of TLR4 is constant in female endometrium (20). Liu et al. showed that LPS-induced endometrial infection played an important part in the occurrence of IUA (2). Therefore, endometrial Klebsiella may be involved in the occurrence of IUA. Further studies are needed to define which Klebsiella species are present in endometrial samples and whether they are capable of inducing pathogenic state.

Furthermore, we found that the proportion of Lactobacillus increased with the severity of adhesion. Although Lactobacillus has long been recognized as the dominant flora of healthy female vagina, not all Lactobacillus is beneficial. It has been proved that most strains of Lactobacillus such as Lactobacillus jensenii, Lactobacillus crispatus, and Lactobacillus gasseri are probiotics (21), while Lactobacillus iners produces less hydrogen peroxide and has weaker ability to resist pathogens (22). Therefore, endometrial Lactobacillus could exist in the micro-ecological imbalance or balance state. In different state, more than $10 \%$ of gene expression of the strain is different, and the expression of related metabolic enzymes increases (23). All the above results suggest that high abundance of Lactobacillus in patients with IUA may contain Lactobacillus iners, which need subsequent isolated culture. Moreover, due to the different structure and microenvironment of uterine cavity and vagina, the distribution or structure of endometrial Lactobacillus may differ from that of vaginal Lactobacillus.

This study has some limitations. First, the sample size may be small, which might cause that we did not detect significant differences for some comparisons. Second, we did not choose healthy women as the control due to ethical restriction, so we were unable to evaluate whether infertile factors could affect endometrial microbiota. Nevertheless, endometrial microbiota should be confined to intrauterine cavity, and the patients with IUA might have other infertility factors. Moreover, this study lacked the inclusion of negative controls because of ethical restriction. With such low abundance microbiota, this type of control is essential.

In conclusion, the uterine cavity is not sterile and actually contains various bacteria. Uterine microbiota are different between patients with IUA and infertility patients without intrauterine lesions and all potential variation of uterine microbes might cause IUA. Maintaining the balance of reproductive tract microbiota could enhance the repair of endometrium after injuries.

\section{Acknowledgments}

We are thankful to our colleagues who provided expertise. Funding: This study was supported by grants from the National Natural Science Foundation of China (Grant No. 81701396), the Fundamental Research Funds for the Central Universities (Grant No. 2018MS83), High-level Hospital Construction Project (Grant No. KJ012019098), and Medical Scientific Research Foundation of Guangdong Province (Grant No. B2019015), and the Science and Technology Program of Guangzhou (Grant No. 201707010326).

\section{Footnote}

Reporting Checklist: The authors have completed the MDAR reporting checklist. Available at http://dx.doi.org/10.21037/ atm-20-2813

Data Sharing Statement: Available at http://dx.doi. org/10.21037/atm-20-2813

Peer Review File: Available at http://dx.doi.org/10.21037/ atm-20-2813

Conflicts of Interest: All authors have completed the ICMJE uniform disclosure form (available at: http://dx.doi. org/10.21037/atm-20-2813). The authors have no conflicts of interest to declare.

Ethical Statement: The authors are accountable for all aspects of the work in ensuring that questions related 
to the accuracy or integrity of any part of the work are appropriately investigated and resolved. This study was conducted in accordance with the Declaration of Helsinki (as was revised in 2013). The study was approved by the Medical Ethics Committees at the Zhujiang Hospital of Southern Medical University (No. 2019-KY-077-01) and informed consent was taken from all the patients.

Open Access Statement: This is an Open Access article distributed in accordance with the Creative Commons Attribution-NonCommercial-NoDerivs 4.0 International License (CC BY-NC-ND 4.0), which permits the noncommercial replication and distribution of the article with the strict proviso that no changes or edits are made and the original work is properly cited (including links to both the formal publication through the relevant DOI and the license). See: https://creativecommons.org/licenses/by-nc-nd/4.0/.

\section{References}

1. Chen G, Liu L, Sun J, et al. Foxf2 and Smad6 CoRegulation of Collagen 5A2 Transcription Is Involved in the Pathogenesis of Intrauterine Adhesion. J Cell Mol Med 2020;24:2802-18.

2. Liu F, Zhu ZJ, Li P, et al. Creation of a female rabbit model for intrauterine adhesions using mechanical and infectious injury. J Surg Res 2013;183:296-303.

3. AAGL Elevating Gynecologic Surgery. AAGL Practice Report: Practice Guidelines on Intrauterine Adhesions Developed in Collaboration With the European Society of Gynaecological Endoscopy (ESGE). Gynecol Surg 2017;14:6.

4. Moreno I, Simon C. Relevance of assessing the uterine microbiota in infertility. Fertil Steril 2018;110:337-43.

5. Fang RL, Chen LX, Shu WS, et al. Barcoded sequencing reveals diverse intrauterine microbiomes in patients suffering with endometrial polyps. Am J Transl Res 2016;8:1581-92.

6. Walther-António MR, Chen J, Multinu F, et al. Potential contribution of the uterine microbiome in the development of endometrial cancer. Genome Med 2016;8:122.

7. Liu Z, Kong Y, Gao Y, et al. Revealing the interaction between intrauterine adhesion and vaginal microbiota using highthroughput sequencing. Mol Med Rep 2019;19:4167-74.

8. Zhao X, Zhao Q, Zhu X, et al. Study on the correlation among dysbacteriosis, imbalance of cytokine and the formation of intrauterine adhesion. Ann Transl Med 2020;8:52.

9. Beraza N. Fibrosis and the intestinal microbiome; a focus on chronic liver disease. Curr Opin Pharmacol 2019;49:76-81.

10. Han MK, Zhou Y, Murray S, et al. Lung microbiome and disease progression in idiopathic pulmonary fibrosis: an analysis of the COMET study. Lancet Respir Med 2014;2:548-56.

11. Patil S, Patel K, Advani J, et al. Multiomic analysis of oral keratinocytes chronically exposed to shisha. J Oral Pathol Med 2019;48:284-9.

12. Bolger AM, Lohse M, Usadel B. Trimmomatic: a flexible trimmer for Illumina sequence data. Bioinformatics 2014;30:2114-20.

13. Schloss PD, Westcott SL, Ryabin T, et al. Introducing mothur: open-source, platform-independent, communitysupported software for describing and comparing microbial communities. Appl Environ Microbiol 2009;75:7537-41.

14. Kopylova E, Navas-Molina JA, Mercier C, et al. OpenSource Sequence Clustering Methods Improve the State of the Art. mSystems 2016;1:e00003-15.

15. Lawley B, Tannock GW. Analysis of $16 \mathrm{~S}$ rRNA Gene Amplicon Sequences Using the QIIME Software Package. Methods Mol Biol 2017;1537:153-63.

16. Cole JR, Wang Q, Fish JA, et al. Ribosomal Database Project: data and tools for high throughput rRNA analysis. Nucleic Acids Res 2014;42:D633-642.

17. Chang Q, Luan Y, Sun F. Variance adjusted weighted UniFrac: a powerful beta diversity measure for comparing communities based on phylogeny. BMC Bioinformatics 2011;12:118.

18. Bengoechea JA, Sa Pessoa J. Klebsiella pneumoniae infection biology: living to counteract host defences. FEMS Microbiol Rev 2019;43:123-44.

19. Clegg S, Murphy CN. Epidemiology and Virulence of Klebsiella pneumoniae. Microbiol Spectr 2016;4. doi: 10.1128/microbiolspec.UTI-0005-2012.

20. Yun BH, Chon SJ, Choi YS, et al. Pathophysiology of Endometriosis: Role of High Mobility Group Box-1 and Toll-Like Receptor 4 Developing Inflammation in Endometrium. PLoS One 2016;11:e0148165.

21. Zhang Z, Lv J, Pan L, et al. Roles and applications of probiotic Lactobacillus strains. Appl Microbiol Biotechnol 2018;102:8135-43.

22. Petrova MI, Reid G, Vaneechoutte M, et al. Lactobacillus iners: Friend or Foe? Trends Microbiol 2017;25:182-91. 
23. Macklaim JM, Fernandes AD, Di Bella JM, et al.

Comparative meta-RNA-seq of the vaginal microbiota and

Cite this article as: Qiu T, Liu L, Zhou H, Sheng H, He Y, Liu M, Cai H. Analysis of endometrial microbiota in intrauterine adhesion by high-throughput sequencing. Ann Transl Med 2021;9(3):195. doi: 10.21037/atm-20-2813 differential expression by Lactobacillus iners in health and dysbiosis. Microbiome 2013;1:12. 

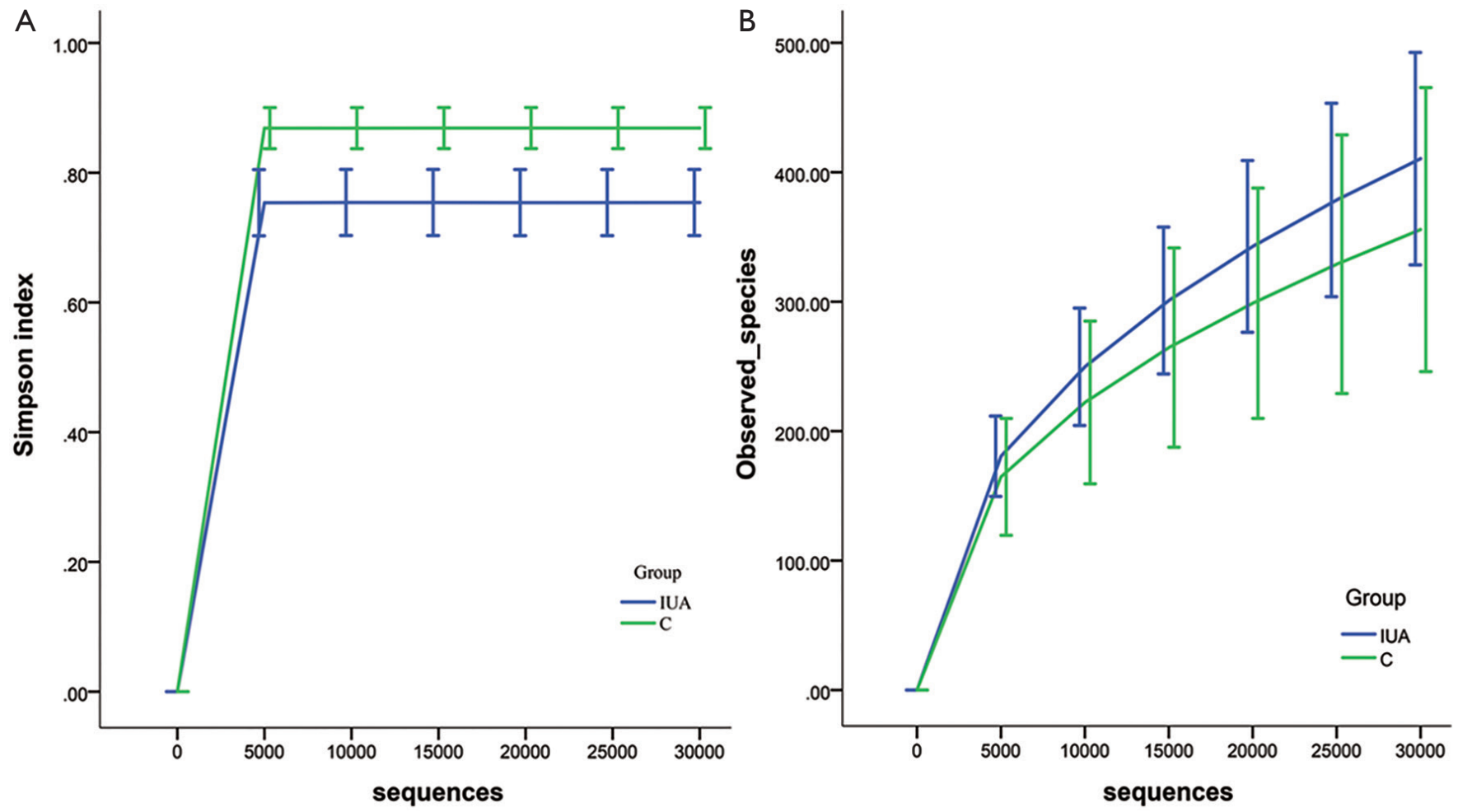

Figure S1 16S rRNA gene analysis indicated lower diversity in patients with IUA and high diversity in infertile females without intrauterine lesion. (A) The rarefaction curves of Simpson index showed that $\alpha$-diversity in patients with IUA was significantly lower than that in infertile females without intrauterine lesion ( $\mathrm{P}=0.01)$. (B) Patients with IUA had more OTUs than infertile females without intrauterine lesion $(\mathrm{P}=0.257)$. These above data were based on $0-30,000$ sequences per $\mathrm{V} 4$ data set.

A

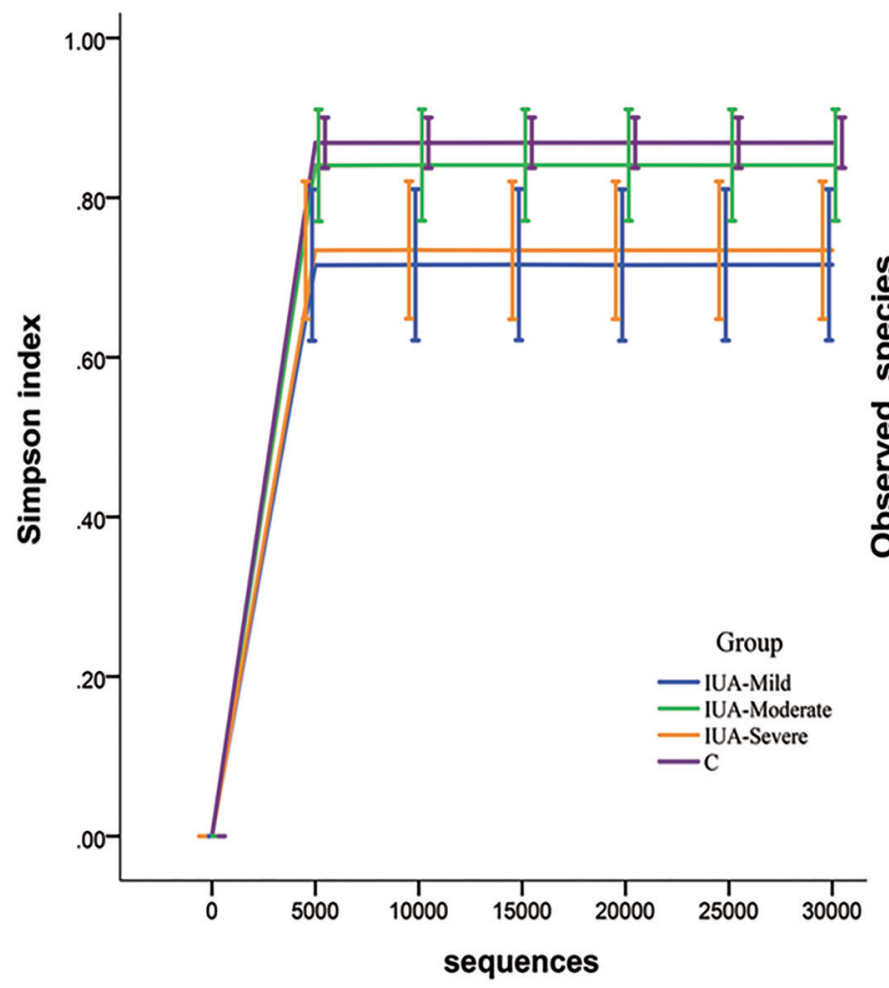

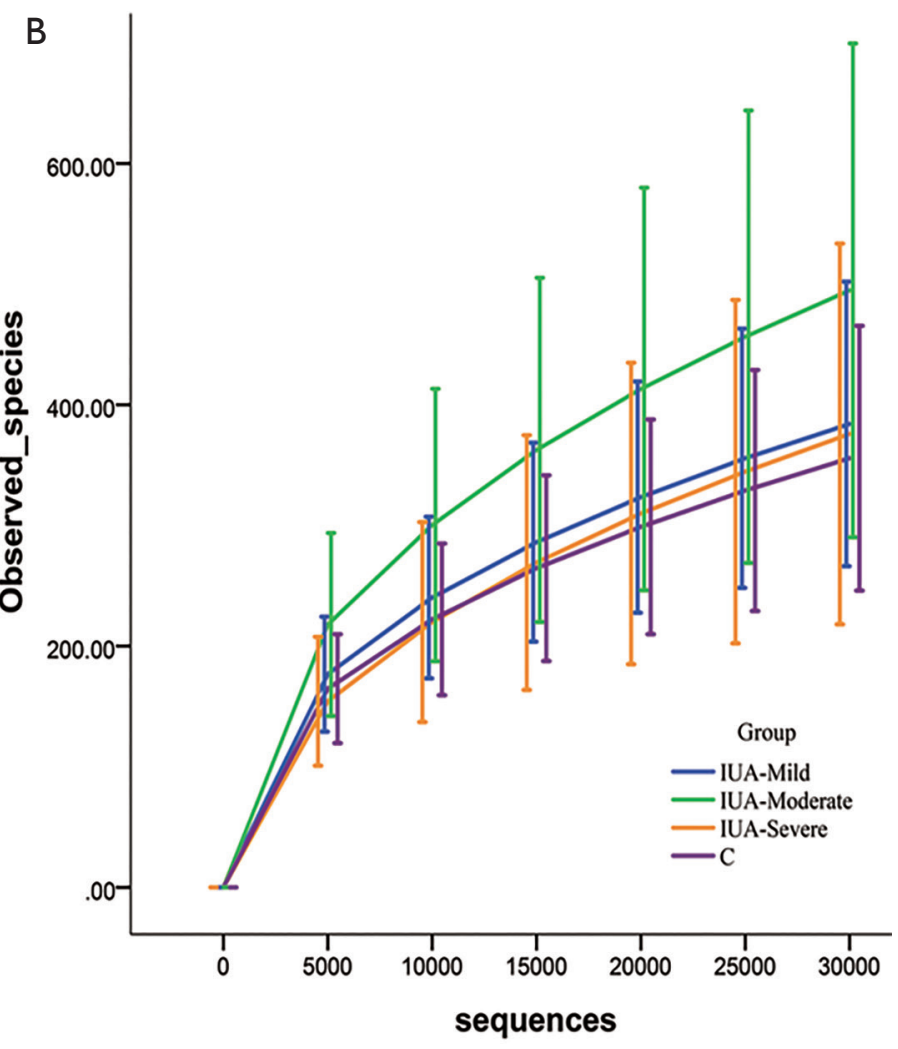

Figure S2 16S rRNA gene analysis indicated different diversities among patients with three IUA subgroups and infertile females without intrauterine lesion. (A) The rarefaction curves of Simpson index indicated significant difference in $\alpha$-diversity of endometrial bacteria between the different stage of IUA and infertile females without intrauterine lesion ( $\mathrm{P}=0.011)$. Infertile females without intrauterine lesion had the highest $\alpha$-diversity of endometrial bacteria. Both endometrial $\alpha$-diversity in the patients with mild IUA and the patients with severe IUA were different from infertile females without intrauterine lesion ( $\mathrm{P}=0.012, \mathrm{P}=0.002)$. (B) There was no difference between the groups in the observed species $(\mathrm{P}=0.491)$. These above data were based on $0-30,000$ sequences per $\mathrm{V} 4$ data set. 
Table S1 Number of raw reads, clean reads and effective in 67 samples of four groups by high-throughput sequencing

\begin{tabular}{|c|c|c|c|c|}
\hline Sample & Raw Reads & Clean Reads & Clean Tags & effective \\
\hline IUA-L1 & 237191 & 236011 & 213819 & 87.22 \\
\hline IUA-L2 & 283199 & 281578 & 267890 & 92.77 \\
\hline IUA-L3 & 292366 & 290654 & 274910 & 91.84 \\
\hline IUA-L4 & 353348 & 351499 & 331449 & 91.21 \\
\hline IUA-L5 & 248050 & 247055 & 230785 & 88.88 \\
\hline IUA-L6 & 144998 & 144332 & 136710 & 90.93 \\
\hline IUA-L7 & 217125 & 216097 & 205840 & 91.57 \\
\hline IUA-L8 & 188815 & 188061 & 177391 & 91.46 \\
\hline IUA-L9 & 228607 & 227467 & 215525 & 92.84 \\
\hline IUA-L10 & 74719 & 74412 & 69150 & 93.77 \\
\hline IUA-L11 & 100024 & 99614 & 92129 & 93.05 \\
\hline IUA-L12 & 66488 & 66277 & 64298 & 97.63 \\
\hline IUA-L13 & 70215 & 69929 & 67585 & 97.34 \\
\hline IUA-L14 & 71556 & 71262 & 69301 & 97.18 \\
\hline IUA-L15 & 116241 & 115842 & 113009 & 97.08 \\
\hline IUA-L16 & 102393 & 101956 & 98495 & 96.95 \\
\hline IUA-L17 & 110076 & 109746 & 106209 & 97.27 \\
\hline IUA-L18 & 91679 & 91356 & 89067 & 97.26 \\
\hline IUA-L19 & 144777 & 144241 & 140720 & 96.99 \\
\hline IUA-L20 & 80983 & 80742 & 77738 & 97.07 \\
\hline IUA-M1 & 273518 & 272098 & 238360 & 90.44 \\
\hline IUA-M2 & 279111 & 277851 & 256228 & 90.28 \\
\hline IUA-M3 & 423955 & 421485 & 398813 & 97.05 \\
\hline IUA-M4 & 347448 & 345274 & 329815 & 93.89 \\
\hline IUA-M5 & 90007 & 89603 & 83026 & 93.08 \\
\hline IUA-M6 & 116856 & 116387 & 105980 & 91.99 \\
\hline IUA-M7 & 172898 & 172145 & 164778 & 92.48 \\
\hline IUA-M8 & 192713 & 191981 & 180111 & 90.26 \\
\hline IUA-M9 & 108764 & 108280 & 100634 & 89.51 \\
\hline IUA-M10 & 124279 & 123808 & 120679 & 96.7 \\
\hline IUA-M11 & 110977 & 110579 & 107642 & 97.01 \\
\hline IUA-M12 & 138066 & 137471 & 133337 & 97.2 \\
\hline IUA-H1 & 317647 & 316049 & 303506 & 96.04 \\
\hline IUA-H2 & 339318 & 337394 & 322330 & 92.58 \\
\hline IUA-H3 & 278663 & 277185 & 261956 & 93.02 \\
\hline IUA-H4 & 264109 & 262650 & 247099 & 85.36 \\
\hline IUA-H5 & 201741 & 200843 & 191721 & 94.78 \\
\hline IUA-H6 & 76573 & 76252 & 72378 & 92.99 \\
\hline IUA-H7 & 57090 & 56869 & 54727 & 92.88 \\
\hline IUA-H8 & 51665 & 51462 & 46613 & 94.71 \\
\hline IUA-H9 & 143699 & 143165 & 139607 & 97.5 \\
\hline IUA-H10 & 93160 & 92825 & 90194 & 97.15 \\
\hline IUA-H11 & 102579 & 102240 & 99942 & 97.78 \\
\hline IUA-H12 & 96858 & 96531 & 94128 & 97.02 \\
\hline IUA-H13 & 93553 & 93240 & 90821 & 97.17 \\
\hline IUA-H14 & 70021 & 69799 & 66932 & 96.69 \\
\hline C1 & 342848 & 340861 & 322066 & 91.44 \\
\hline C2 & 246925 & 245707 & 232991 & 87.84 \\
\hline C3 & 209052 & 207954 & 199902 & 94.34 \\
\hline C4 & 296978 & 295557 & 281284 & 89.82 \\
\hline C5 & 163580 & 162992 & 154773 & 91.78 \\
\hline C6 & 144029 & 143427 & 136028 & 89.8 \\
\hline $\mathrm{C} 7$ & 100138 & 99748 & 92847 & 94.03 \\
\hline C8 & 58560 & 58301 & 54959 & 91.84 \\
\hline C9 & 183039 & 182294 & 174081 & 94.13 \\
\hline C10 & 103415 & 102964 & 94463 & 91.72 \\
\hline C11 & 48172 & 47952 & 39339 & 94.03 \\
\hline C12 & 63169 & 62960 & 60356 & 96.77 \\
\hline C13 & 67383 & 67144 & 65398 & 97.01 \\
\hline C14 & 72544 & 72324 & 69459 & 96.87 \\
\hline C15 & 66409 & 66157 & 63548 & 96.9 \\
\hline C16 & 129163 & 128678 & 124356 & 97.39 \\
\hline C17 & 115265 & 114905 & 111477 & 97.44 \\
\hline C18 & 93204 & 92875 & 89512 & 97.51 \\
\hline C19 & 111262 & 110869 & 107899 & 94.32 \\
\hline C20 & 157915 & 157347 & 152031 & 97.77 \\
\hline C21 & 123292 & 122809 & 119509 & 97.13 \\
\hline Total & 10684460 & 10635422 & 10089655 & I \\
\hline Average & 159469.55 & 158737.64 & 150591.9 & 94.12 \\
\hline
\end{tabular}

IUA-L, intrauterine adhesion patients with low-grade; IUA-M, intrauterine adhesion patients with middle-grade; IUA-H, intrauterine adhesion patients with high-grade; C, control group. 